\title{
Diagnostic Accuracy of Glycated Haemoglobin and Average Glucose Values in Type 2 Diabetes Mellitus Treated with Premixed Insulin
}

\author{
Magdalena Walicka · Jacek Jozwiak · Jacek Rzeszotarski • \\ Anna Zonenberg • Malgorzata Masierek • Pawel Bijos • \\ Edward Franek
}

Received: October 23, 2018 / Published online: February 8, 2019

(c) The Author(s) 2019

\section{ABSTRACT}

Introduction: Studies assessing the relationship between glycated haemoglobin (HbA1c) and average blood glucose (ABG) were conducted in small groups of patients on different treatments and may be biased for these reasons. The aim of the study was to assess the relationship between $\mathrm{HbA} 1 \mathrm{c}$ and $\mathrm{ABG}$ in a large group of type 2 diabetes patients treated with premix insulin.

Methods: In 4257 patients treated with premixed insulin, the parallel point-of-care assessment of HbA1c and ABG from the preceding 90 days (ABG90), calculated automatically from

Enhanced Digital Features To view enhanced digital features for this article go to https://doi.org/10.6084/ m9.figshare.7593974.

M. Walicka · E. Franek ( $\square)$

Department of Internal Diseases, Endocrinology and

Diabetology, Central Clinical Hospital of the

Ministry of the Inferior and Administration,

Warsaw, Poland

e-mail: edward.franek@cskmswia.pl

J. Jozwiak

Department of Public Health, Czestochowa

University of Technology, Czestochowa, Poland

J. Jozwiak

Silesian Analytical Laboratories, Katowice, Poland

J. Rzeszotarski

Clinical Department of Internal Diseases and

Diabetology, 10th Military Hospital, Bydgoszcz,

Poland all values measured by the glucometer, was performed twice. The regression formulas and respective values of $\mathrm{HbA} 1 \mathrm{c}$ and $\mathrm{ABG} 90$ were calculated.

Results: The mean number of recorded glucose values/patient was 2.37 estimations per day. The regression formula calculated using data from the first assessment was HbA1c $=5.28+$ $0.01487 \times$ ABG90 and that using data from the second one was HbA1c $=4.78+0.01683 \times$ ABG90. The slopes of the regression lines are lower than that in a similar analysis from the A1c-Derived Average Glucose (ADAG) study. The comparison of ADAG formula and the formula derived from the present study shows that both formulas give similar results at low HbA1c values, but differ at higher HbA1c values. Additionally, the 95\% confidence interval is

\section{A. Zonenberg}

Medical Institute, Higher School of Computer

Science and Business Administration, Lomza,

Poland

M. Masierek

Bioton SA, Warsaw, Poland

P. Bijos

Private Practice, Warsaw, Poland

E. Franek

Department of Human Epigenetics, Mossakowski

Medical Research Centre, Polish Academy of

Sciences, Warsaw, Poland 
broader in the PROGENS study e.g. a 95\% probability of certainty that the actual HbA1c value was greater than $7.0 \%(53 \mathrm{mmol} / \mathrm{mol})$ was achieved only at an ABG90 value of $220 \mathrm{mg} / \mathrm{dL}$. Conclusion: The relationship between HbA1c and $A B G$ estimations may be different in various patients; therefore, it seems that the use of one equation in all populations may not be reliable. Broad assessment of $A B G$ as a tool that may replace HbA1c measurements should be recommended only with caution, providing the possible limitations and confidence intervals.

Funding: Bioton S.A.

Keywords: Average blood glucose; Glycated haemoglobin; HbA1c; Premixed insulin; Type 2 diabetes

\section{INTRODUCTION}

Glycated haemoglobin (HbA1c) is used for assessing diabetes management and more recently also for the diagnosis of diabetes [1-3]. However, it is associated with some limitations [4-7] and its use in many countries, including the USA [8], is limited. The reasons are mainly financial, but logistical reasons, like no access to laboratories, may also play some role. It is also worth noting that HbA1c does not inform patients about blood glucose values on a daily basis and some patients do not understand what HbA1c means.

Therefore, in 2009, shortly after the publication of the A1c-Derived Average Glucose (ADAG) study [9] that provided data allowing the estimation of average blood glucose (ABG) values from HbA1c values, the American Diabetes Association (ADA) incorporated those data while framing the guidelines [10]. This practical algorithm, shown in the form of a table, may serve "as a check on the accuracy of the patient's meter" [11]. However, in practice, it can also be used for reverse calculation, i.e. for estimating the HbA1c value from the ABG values and for assessing whether the therapeutic targets have been met in those patients for whom HbA1c measurement is not possible.

In clinical practice, however, there may be differences between the actual, measured
HbA1c and the calculated value. In such cases, it is often argued that patients do not record high glucose values in their diaries, and therefore the calculations may be biased. Although it may be true in many cases, this explanation would apply only in the situation where a high HbA1c level is matched with a low ABG, whereas the opposite situation is also observed. It seems, therefore, that there is some biological variability that may influence glycated haemoglobin. Results of some studies suggest that a substantial portion of HbA1c is determined by nonglycemic factors [12]. Further, certain conditions, e.g. anaemia, rheumatoid arthritis and chronic kidney disease, could have influenced the results of HbA1c.

Studies evaluating the relationship between blood glucose and haemoglobin A1c levels in subjects with diabetes have some limitations. The Irish study was performed in a relatively small group of patients [13]. A similar study, based on the Diabetes Control and Complications Trial (DCCT) data, was performed only in type 1 diabetes patients and the results differ from the results of the ADAG study in that higher $A B G$ values were calculated from the respective HbA1c values, and the results had a broader distribution, at least at higher HbA1c and glucose values [14]. In the ADAG study [9] only 159 had type 2 diabetes. Of the remaining subjects, 80 had normal glucose tolerance (NGT) with low glucose and HbA1c values. The data from the subjects with NGT (low HbA1c and low average glucose) could affect the slope of the regression line. Additionally type 2 diabetes patients were an average of 15 years older than those with NGT and 11 years older than patients with type 1 diabetes and certain conditions in elderly, multimorbid patients with type 2 diabetes conditions could have influenced the results. Moreover, the average glucose value in the ADAG study was calculated by combining weighted results from the continuous glucose monitoring (CGM) performed every 4 weeks (over at least a 48-h period, with glucose levels assessed every $5 \mathrm{~min}$ ) with 8-point daily selfmonitoring of capillary (fingerstick) glucose performed at least every 2 days, or in patients not on CGM, 7-point daily monitoring for 3 days a week during a 12-week study period. 
This is definitely not a real-life situation. Normally the number of records does not exceed 4 values per day, whereas nocturnal values are only rarely recorded. Thus, in common practice, the calculations are based on a few values captured each day rather than many values captured over greater time intervals. Another study used only fasting plasma glucose values, achieving results similar to those of the ADAG study [15].

A new analysis of the ADAG Study Group report empirically links target blood glucose values with HbA1c levels [16]. The authors calculated the average fasting, postprandial and bedtime self-monitored blood glucose concentrations associated with specified HbA1c levels achieved by subjects in the ADAG study. The relationship between $\mathrm{HbA1c}$ and fasting glucose may be different from its relationship with postprandial glucose [13, 17]. A recently published systematic review and meta-analysis found that postprandial glucose has a closer association with $\mathrm{HbA1c}$ than fasting glucose (although the heterogeneity score was high) [18]. In a widely cited study by Monnier, the relative contribution of postprandial glucose excursions was predominant in fairly controlled patients, whereas the contribution of fasting hyperglycaemia increased gradually with diabetes worsening [19].

Taking into account the above facts, as the reviewed trials show some high-quality evidence but have also some limitations, we have only moderate confidence that a relationship between $\mathrm{HbA1c}$ is adequately established. It is, for example, not clear whether the results are equally reliable in patients with type 2 as with type 1 diabetes. Additionally, studies on larger groups are needed. Thus this study aimed to assess the relationship between HbA1c and average glucose concentrations in a large number of type 2 diabetes patients treated with premixed insulin.

\section{METHODS}

\section{Study Design and Participants}

This study is a pre-specified analysis of the data from the PROGENS HbA1c study [20], a phase 4 observational study, in which 4257 subjects who started insulin treatment with premixed human insulin (Gensulin, Bioton, Poland) were observed for 6 months. The primary aim was to assess the effectiveness and safety of this drug, and the secondary aim was to compare the HbA1c value with the ABG value. The protocol of the study was prepared according to the Declaration of Helsinki and approved by the appropriate ethics committee. All subjects signed an informed consent document.

The parallel assessment of HbA1c and average blood glucose from the preceding 90 days (ABG 90 days) was performed at two visits (visit 2 and 3). All subjects were supplied with a Glucocard 01-Mini Plus glucometer (Arkray, Kyoto, Japan). In accordance with the guidelines of the Polish Diabetes Association [21], subjects were instructed to monitor glucose value every day once or twice, if the dose of premixed insulin was stable, and additionally at least once a week in a 4-point blood glucose profile (fasting and after every main meal). In patients in whom insulin dose was changed (per protocol in poorly controlled patients at visit 1 or 2), more intensive control was advised with an additional 7-point glycaemic profile (fasting, before and after every main meal) at least once a week during 2 or 3 weeks, depending on the glucose values. The average blood glucose was calculated automatically from all values measured by the glucometer in the 90 days preceding visits 2 and 3 . Therefore, in contrast to studies conducted using diaries where patients may not note some results, every measured result was included in the analysis.

The HbA1c in the capillary blood was measured using the multitest HbA1c analyser A1cNow+ (Bayer HealthCare, Germany), which is a certified method listed in the National Glycated Haemoglobin Standardisation Program (NGSP) [22]. The staff at each study centre were appropriately trained to perform the procedure. Additionally, in order to monitor the quality of HbA1c measurements in the local settings, an additional analysis was performed in 120 patients (six centres, 15 patients in each; one centre, 30 patients) with parallel high-performance liquid chromatography (HPLC), performed in a central laboratory. At the time of 
capillary blood collection, venous blood samples $(2-4 \mathrm{~mL}$ of blood with anticoagulant ethylenediaminetetraacetic acid) were taken. Blood samples were transported by laboratory couriers (at $4-8{ }^{\circ} \mathrm{C}$ in electronically controlled containers) to the central facility where all analyses were conducted within $3 \mathrm{~h}$ of blood collection. All HbA1c measurements were conducted in the same International Organization for Standardization (ISO)-certified laboratory using an automated bio-analyzer (BIO-RAD VARIANT II TURBO Hemoglobin Testing System, Bio-Rad Laboratories, USA); the HPLC method is listed as an NGSP certified method [22]. HbA1c concentrations were measured using the VARIANT II TURBO HbA1c Kit-2.0 (Elution Buffer A and Elution Buffer B). The performing staff were blinded to the A1cNow+ device results.

\section{Statistical Analyses}

Statistical regression analysis was performed for assessing the association between quantitative variables. The relationship is presented in an analytical form and graphically by means of a regression line with a 95\% confidence interval. The analytical form of the linear regression was used to estimate the mean plasma glucose as a function of HbA1c levels, and a logistic regression was used for approximation of probability of high HbA1c values (above 7.0\%, $53 \mathrm{mmol} / \mathrm{mol}$ ). Correlation between $\mathrm{HbA} 1 \mathrm{c}$ values measured by HPLC and by the A1cNow+ device was expressed using Pearson's correlation coefficient. Patient baseline data and biochemical measurements are presented as mean (SD). Analyses were performed using the SAS System (9.3).

\section{RESULTS}

\section{Baseline Demographic and Clinical Features}

Data on both HbA1c and ABG were available for 3416 patients at visit 2 and for 3331 patients at visit 3. Baseline data of the patients are shown
Table 1 Data for 3416 patients at visit 2 and for 3331 patients at visit 3 for whom both the HbAlc and ABG data were available (I) and the data for 120 patients (63 women and 57 men) in whom HPLC was performed (II)

\begin{tabular}{lll}
\hline & I & II \\
\hline Mean age (years) & $63.7( \pm 9.4)$ & $63.5( \pm 9.2)$ \\
Height $(\mathrm{cm})$ & $168( \pm 9)$ & $168( \pm 8)$ \\
Weight $(\mathrm{kg})$ & $85.6( \pm 14.7)$ & $83.5( \pm 16.3)$ \\
BMI $\left(\mathrm{kg} / \mathrm{m}^{2}\right)$ & $30.3( \pm 4.5)$ & $29.6( \pm 5.2)$ \\
$\begin{array}{l}\text { Fasting plasma glucose } \\
\quad(\mathrm{mg} / \mathrm{dl})\end{array}$ & $151( \pm 39)$ & $144( \pm 27)$ \\
$\begin{array}{l}\text { Diabetes duration (years) } \\
\text { Serum creatinine }(\mathrm{mg} / \mathrm{dl})\end{array}$ & $9.0( \pm 5.5)$ & $10.3( \pm 6.4)$ \\
Haemoglobin $(\mathrm{Hb})$ & $13.7( \pm 1.5)$ & $0.93( \pm 0.5)$ \\
$\quad(\mathrm{g} / \mathrm{dl})$ & & \\
\hline
\end{tabular}

Data are presented as mean $(\mathrm{SD})$

in Table 1. The mean number of recorded glucose values/patient was 214 in 90 days (2.37 estimations per day). On average 104 estimations were made postprandially, 98 before breakfast (fasting) and 12 before other meals. The postprandial estimations were performed approximately $2 \mathrm{~h}$ after breakfast.

\section{Correlation Between HbA1c Values and ABG 90 Days}

Hyperglycaemia improved significantly during the study; the mean HbA1c at visits 1, 2 and 3 was $8.3 \%( \pm 1.2 \%)(67 \mathrm{mmol} / \mathrm{mol}), 7.8 \%( \pm 0.9)$ $(62 \mathrm{mmol} / \mathrm{mol})$ and $7.4 \% \quad( \pm \quad 0.8)$ $(57 \mathrm{mmol} / \mathrm{mol})$, respectively. The average daily glucose at visits 2 and 3 was $166( \pm 31) \mathrm{mg} / \mathrm{dL}$ and $155( \pm 24) \mathrm{mg} / \mathrm{dL}$, respectively.

The regression formula calculated using data from the first assessment was HbA1c $=5.28+$ $0.01487 \times$ ABG90 and that using data from the second one was $\mathrm{HbA1c}=4.78+0.01683 \times$ ABG90. The formulas and regression lines are similar, although the regression line at visit 3 begins at lower HbA1c and ABG levels, because the values decreased during the study. 
Table 2 Prediction of average blood glucose (ABG) from the HbAlc level in the PROGENS study (visit 2, ABG 90 days $=14.9 \times \mathrm{HbAlc}+45.1)$ and in the ADAG study (calculated $\left.\mathrm{AG}_{\mathrm{mg} / \mathrm{dL}}=28.7 \times \mathrm{HbAlc}(\%)-46.7\right)$

\begin{tabular}{llllll}
\hline $\begin{array}{l}\text { HbA1c } \\
(\%)\end{array}$ & $\begin{array}{l}\text { HbAlc } \\
(\mathbf{m m o l} / \mathbf{m o l})\end{array}$ & $\begin{array}{l}\text { Average glucose } \\
(\text { ADAG })\end{array}$ & $\begin{array}{l}\text { 95\% CI } \\
(\text { ADAG })\end{array}$ & $\begin{array}{l}\text { Average glucose } \\
(\text { PROGENS HbA1c) }\end{array}$ & $\begin{array}{l}\text { 95\% CI (PROGENS } \\
\text { HbA1c) }\end{array}$ \\
\hline 5.0 & 31 & 97 & $76-120$ & & \\
6.0 & 42 & 126 & $100-152$ & 138 & $86-190$ \\
7.0 & 53 & 154 & $123-185$ & 154 & $103-206$ \\
8.0 & 64 & 183 & $147-217$ & 170 & $119-222$ \\
9.0 & 75 & 212 & $170-249$ & 187 & $135-238$ \\
10.0 & 86 & 240 & $193-282$ & 203 & $151-254$ \\
11.0 & 97 & 269 & $217-314$ & 219 & $167-271$ \\
12.0 & 108 & 298 & $240-347$ & 235 & $183-287$ \\
13.0 & 119 & & & 251 & $188-292$ \\
\hline
\end{tabular}

Table 2 shows the calculation of ABG from HbA1c values at visit 2, using both the ADAG formula [9] and the formula derived from the present study. The comparison shows that both formulas give identical results at HbA1c value equal 7\% (although the 95\% confidence interval is broader in the PROGENS study), but differ at higher $\mathrm{HbA1c}$ values ( $8 \%$ or more) and the difference increases with increasing HbA1c. Table 3 shows how the formula used for calculating $A B G$ from $\mathrm{HbA1c}$ can be used in reverse, for calculating HbA1c from $A B G$ values. In the last column of Table 3 , a probability of the actual HbA1c value being greater than $7.0 \%$ $(53 \mathrm{mmol} / \mathrm{mol})$ (i.e. that a patient has not reached the treatment target) was calculated on the basis of the ABG values.

\section{Correlation Between Two Methods for Measuring HbA1c in the PROGENS Study: HPLC and Multitest HbA1c Analyser Ac1Now+}

The subset of 120 patients in whom HPLC was performed comprised 63 women and 57 men. Baseline data of the patients are shown in Table 1. Those values were comparable with the baseline data of the whole population included in the PROGENS HbA1c study. The mean HbA1c measured by HPLC was 7.6\% $( \pm 1.6 \%)$
(60 $\mathrm{mmol} / \mathrm{mol})$, and that by the multitest A1cNow+ was $7.5 \%( \pm 1.4 \%)(59 \mathrm{mmol} / \mathrm{mol})$ (mean difference, $0.059( \pm 0.647)$; median difference, 0 ; minimum, -1.7 ; maximum, +1.6 ). The difference was greater than 1 in 10 patients and 0 in 12 patients. In one patient, the A1cNow+ HbA1c estimation was not performed. The correlation between the two methods of HbA1c measurement is shown in Fig. 1.

\section{DISCUSSION}

This study was aimed at assessing the relationship between HbA1c and average glucose concentrations in a large number of type 2 diabetes patients, as this is the most prevalent type of diabetes, and the calculation of ABG from HbA1c (and vice versa) is more useful in type 2 diabetes (in intensively treated type 1 diabetes patients usually both HbA1c and blood glucose are measured and accessible; therefore, such calculation is not necessary). The results were compared with the results of the ADAG study [9], which is the most widely cited and most widely used for the calculation of ABG from HbA1c. We found that use of HbA1c as a tool to enable average glucose estimations or to check the accuracy of a glucometer may be not as 
Table 3 Prediction of HbAlc level from the average plasma glucose in the preceding 90 days (ABG 90 days) in patients with type 2 diabetes $(n=4257)$ and the estimated probability that the actual HbAlc value is greater than $7 \%$, depending on the $A B G$ value

\begin{tabular}{llll}
\hline ABG 90 days & Predicted HbA1c level (\%) & $\mathbf{9 5 \%} \mathbf{C I}$ & Probability of HbA1c > 7.0\% (95\% CI) \\
\hline 100 & 6.5 & $5.1-7.9$ & $0.22(0.18-0.25)$ \\
110 & 6.6 & $5.2-8.0$ & $0.28(0.25-0.32)$ \\
120 & 6.8 & $5.4-8.2$ & $0.36(0.33-0.39)$ \\
130 & 7.0 & $5.6-8.4$ & $0.44(0.41-0.47)$ \\
140 & 7.1 & $5.8-8.5$ & $0.53(0.51-0.55)$ \\
150 & 7.3 & $5.9-8.7$ & $0.61(0.60-0.63)$ \\
160 & 7.5 & $6.1-8.9$ & $0.69(0.67-0.71)$ \\
170 & 7.6 & $6.3-9.0$ & $0.76(0.74-0.78)$ \\
180 & 7.8 & $6.4-9.2$ & $0.82(0.80-0.84)$ \\
190 & 8.0 & $6.6-9.4$ & $0.87(0.84-0.88)$ \\
200 & 8.2 & $6.8-9.5$ & $0.90(0.88-0.92)$ \\
210 & 8.3 & $6.9-9.7$ & $0.93(0.91-0.94)$ \\
220 & 8.5 & $7.1-9.9$ & $0.95(0.93-0.96)$ \\
230 & 8.7 & $7.3-10.0$ & $0.96(0.95-0.97)$ \\
240 & 8.8 & $7.4-10.2$ & $0.97(0.96-0.98)$ \\
250 & 9.0 & $7.6-10.4$ & $0.98(0.97-0.99)$ \\
260 & 9.2 & $7.8-10.6$ & $0.99(0.98-0.99)$ \\
\hline
\end{tabular}

Data from visit 2 are shown

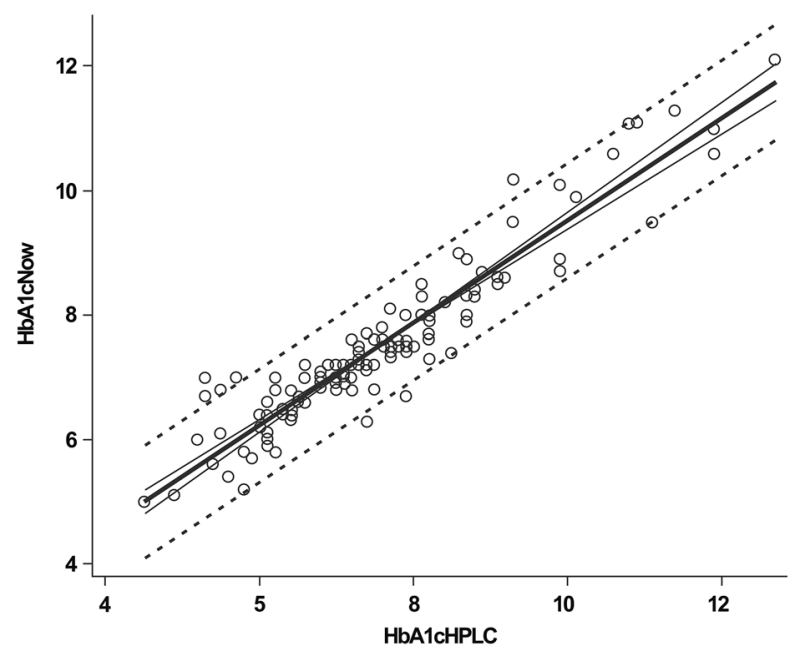

Fig. 1 Correlation between the two methods for measuring HbAlc in the PROGENS study: HPLC and multitest HbAlc analyser Ac1Now+ 
reliable as suggested by the ADAG study and ADA guidelines.

The comparisons of $A B G$ calculation from HbA1c (Table 2) using both the ADAG formula [9] and the formula derived from the present study begin and end at different values. This is because the ADAG study included healthy subjects with low $\mathrm{HbA1c}$ and $\mathrm{ABG}$ values.

In the ADAG study the data from the subjects with NGT (low HbA1c and low average glucose) could affect the slope of the regression line. Additionally, it was not clear whether and how the HbA1c values in those groups overlapped; in other words, whether and how many subjects with NGT had HbA1c values higher than the upper limit of the normal range, or how many diabetic subjects had well-controlled disease with low HbA1c. The PROGENS study included only type 2 diabetes patients, which explains the absence of low and the presence of higher $A B G$ and HbA1c values. The comparison shows that both formulas give similar results at low HbA1c values (although the 95\% confidence interval is broader in the PROGENS study), but differ at higher HbA1c values. However, the slope of the regression lines would not be as steep. This means that even though the regression formulas in the ADAG and PROGENS studies give similar results at the lower range of $\mathrm{HbA1c}$ values $(6.0-8.0 \%)(42-64 \mathrm{mmol} / \mathrm{mol})$, the predicted ABG values at the higher range of $\mathrm{HbA1c}$ $(9.0-12.0 \%)(75-108 \mathrm{mmol} / \mathrm{mol})$ will be overestimated with the ADAG formula for our population. In fact, the predicted $A B G$ values for our population in that range were more than $60 \mathrm{mg} /$ dL higher when calculated by the ADAG formula (Table 2). This result is probably caused by differences in $\mathrm{HbA} 1 \mathrm{c}$ measurements between the point-of-care and HPLC methods. It also calls into question the reliability of using the ADAG formula in patients with type 2 diabetes, in whom point-of-care devices are used, at least in the higher range of HbA1c. Because higher HbA1c values are common in patients with type 2 diabetes, this discrepancy may be a significant practical issue.

The distribution of the results in the present study is broader than in the ADAG study. This is due to a much higher number of participants and other reasons discussed in the
"Introduction". The broader distribution and the wider 95\% confidence interval may influence the reliability of the calculation. Unfortunately, the ADA guidelines do not show the 95\% confidence intervals in the table showing the $A B G$ values calculated from the given HbA1c values [11], which suggests to practitioners that the calculation is entirely reliable.

In many countries and centres where the access to reliable HbA1c measurement is limited, it is much more common to use the reverse calculation, i.e. from ABG to HbA1c, as shown in Table 3. Translating average glucose values to HbA1c may be a practical solution for those physicians and endocrinologists. However, this study raises some concerns regarding the use of $\mathrm{ABG}$ as a tool for patient assessment. For example, the 95\% confidence intervals for the calculation of HbA1c from various glucose levels (as shown in Table 3) are broad over the entire range of glucose values. The probability of a real patient's HbA1c being over the treatment target for a given $A B G$ value is shown in the last column of Table 3 . As can be seen, relying only on the estimated HbA1c value makes the probability of inaccurate judgements rather high. In other words, there is a high probability that a physician trying to assess HbA1c from ABG (and therefore trying to assess whether the patient's diabetes is well controlled) may be in error. An ABG value as high as $220 \mathrm{mg} / \mathrm{dL}$ or more is required to achieve a 95\% probability that the patient's actual HbA1c value is over the treatment target (i.e. greater than $7.0 \%, 53 \mathrm{mmol} / \mathrm{mol}$ ). At the same time, there is only a $72 \%$ probability that a patient with an ABG of $110 \mathrm{mg} / \mathrm{dL}$ has an actual HbA1c value of $7.0 \%(53 \mathrm{mmol} / \mathrm{mol})$ or less. In other words, in almost one third of the patients with an $A B G$ of $110 \mathrm{mg} / \mathrm{dl}$, the actual HbA1c value is over the general therapeutic target. These data highlight the unreliability of this type of assessment. Unfortunately, similar evaluations were not performed in the other studies assessing the relationship between ABG and HbA1c.

The differences between the results obtained in our study and the ADAG study may be due to various reasons. The ADAG study have used standard A1C estimation, whereas in the present study point-of-care A1C is used. The 
differences may also be due to the different accuracies of the glucometers. It also could be due to the different number of values that had been done in fasting or prandial state. However, it should be emphasized that such differences are also present in real life.

We tested whether the HbA1c values measured by the point-of-care device were reliable, using a direct side-by-side comparison with the gold standard HPLC method. Some studies comparing HbA1c point-of-care test results with those from laboratory analyses suggest no significant differences between the methods [23-25], but others suggest that proficiency testing should mandatory for users of HbA1c pointof-care assays to ensure quality [26]. The HbA1c analyser A1cNow+ method is included in the NGSP list, and the method is continuously validated. Our study in the local setting also confirmed the correlation between this method and the gold standard HPLC method (Fig. 1); however, the correlation line is shifted in the higher range of haemoglobin values. This difference implies that the real HbA1c may be higher than that measured by the point-of-care device. This is not of importance from a practical viewpoint, as regardless of whether the real HbA1c is $10 \%$ or $12 \%$, it is well above the target and the patient requires intensification of treatment. However, it seems that if the line had not been shifted, the slope of the regression line in the ADAG and PROGENS studies would have been similar.

The study has some limitations. First, pointof-care devices were used for assessing glucose and HbA1c, and the results may therefore be different had golden standard methods been used. However, point-of-care devices or other non-laboratory methods are used for HbA1c estimation in clinical practice. Use of point-ofcare devices reflects the practice in real-life settings. In addition, only one kind of glucometer was used at all sites, ensuring the comparability of the results. With regard to HbA1c estimation, as described above and shown in Fig. 1, the correlation with HPLC estimations is very good.

The examined population (although numerous, which may be regarded as a strength of the study) is limited to premixed insulintreated patients with type 2 diabetes. This may be regarded as another limitation of the study.
Indeed, it is not known whether and how the treatment may influence the relationship between HbA1c and ABG. Therefore caution is required when translating the results to other populations.

There are multiple mechanisms underlying the variations in $\mathrm{HbA1c}$, blood glucose and the relationship between them $[5,6,27]$. Genetics are thought to play a significant role in the determination of $\mathrm{HbA}_{1 \mathrm{c}}$ [28]. $\mathrm{HbA}_{1 \mathrm{c}}$ levels are genetically determined, with heritability of 47-59\% [29]. In Jansen's study [12] age, gender, BMI, FPG, mean corpuscular haemoglobin, mean corpuscular haemoglobin concentration, current smoking and alcohol consumption were independent predictors of HbA1c, together explaining $26.2 \%$ of the variance in HbA1c, with FPG contributing $10.9 \%$. Rather than assuming that it is possible to make precise calculations based on the results of this study, the ADAG study or other formulas, we suggest that such calculations should be seen as only rough assessments of glycaemic control. As the $\mathrm{ABG}$ is used more and more frequently, especially in those countries or regions where HbA1c tests are too expensive, inaccessible or both, it is important that physicians be aware of the limitations of this method, in terms of calculation the HbA1c from ABG. In parallel, use of HbA1c for assessing ABG or checking accuracy of a glucometer may also be unreliable.

\section{CONCLUSIONS}

The relationship between HbA1c and ABG estimations may be different in various patients; therefore, it seems that the use of one equation in all populations may not be reliable. Broad assessment of $\mathrm{ABG}$ as a tool that may replace HbA1c measurements should be recommended only with caution, providing the possible limitations and confidence intervals.

\section{ACKNOWLEDGEMENTS}

Funding. The PROGENS HbA1c study and the journal's article processing charges were 
funded by Bioton S.A. The funding source had no role in interpretation of the data, or preparation of the manuscript. All authors had full access to all of the data in this study and take complete responsibility for the integrity of the data and accuracy of the data analysis. The authors had complete control over the interpretation of the results and writing of the manuscript.

Authorship. All named authors meet the International Committee of Medical Journal Editors (ICMJE) criteria for authorship for this article, take responsibility for the integrity of the work as a whole, and have given their approval for this version to be published.

Disclosures. Malgorzata Masierek: Employee, Bioton S.A. Pawel Bijos: former employee, Bioton S.A. Edward Franek: Advisory Panel-Boehringer Ingelheim, Eli Lilly, Novo. Research Support-Bayer, Bioton. Speaker's Bureau-AstraZeneca, Bioton, Boehringer Ingelheim, Eli Lilly, MSD, Novo, Polfa Tarchomin, Servier. Magdalena Walicka, Jacek Jozwiak, Jacek Rzeszotarski and Anna Zonenberg have nothing to disclose.

Compliance with Ethics Guidelines. All procedures performed in studies involving human participants were in accordance with the ethical standards of the institutional and/or national research committee and with the 1964 Helsinki declaration and its later amendments or comparable ethical standards. Informed consent was obtained from all individual participants included in the study. The authors thank all the participants of the study.

Data Availability. The datasets obtained and/or analyzed during the current study are available from the corresponding author on reasonable request.

Open Access. This article is distributed under the terms of the Creative Commons Attribution-NonCommercial 4.0 International License (http://creativecommons.org/licenses/ by-nc/4.0/), which permits any noncommercial use, distribution, and reproduction in any medium, provided you give appropriate credit to the original author(s) and the source, provide a link to the Creative Commons license, and indicate if changes were made.

\section{REFERENCES}

1. The International Expert Committee. International Expert Committee report on the role of the A1c assay in the diagnosis of diabetes. Diabetes Care. 2009;32:1327-34.

2. World Health Organization Consultation. Use of glycated haemoglobin (HbA1c) in the diagnosis of diabetes mellitus. Diabetes Res Clin Pract. 2011;93:299-309.

3. American Diabetes Association. Classification and diagnosis of diabetes. Diabetes Care. 2017;40(Suppl1):S11-24.

4. Lippi G, Targher G. Glycated hemoglobin (HbA1c): old dogmas, a new perspective? Clin Chem Lab Med. 2010;48:609-14.

5. Sacks DB, Arnold M, Bakris GL, et al. National Academy of Clinical Biochemistry. Position statement executive summary: Guidelines and recommendations for laboratory analysis in the diagnosis and management of diabetes mellitus. Diabetes Care. 2011;34:1419-23.

6. Sacks DB. Measurement of HbA1c. A new twist on the patch to harmony. Diabetes Care. 2012;35:2674-80.

7. Shepard JG, Airee A, Dake AW, McFarland MS, Vora A. Limitations of A1c interpretation. South Med J. 2015;108:724-9.

8. Liao Y, Bang D, Cosgrove S, et al. Surveillance of health status in minority communities: Racial and Ethnic Approaches to Community Health Across the US (REACH U.S.) Risk Factor Survey, US 2009. MMWR Surveill Summ. 2011;60:1-44.

9. Nathan DM, Kuenen J, Borg R, Zheng H, Schoenfeld D, Heine RJ. A1c-Derived Average Glucose Study Group. Translating the A1c assay into estimated average glucose values. Diabetes Care. 2008;31:1473-1478.

10. American Diabetes Association. Standards of medical care in diabetes-2009. Diabetes Care. 2009;32(Suppl. 1):S13-61.

11. American Diabetes Association. Glycemic targets. Diabetes Care. 2017;40(Suppl 1):S48-56. 
12. Jansen H, Stolk RP, Nolte IM, Kema IP, Wolffenbuttel $\mathrm{BH}$, Snieder H. Determinants of HbA1c in nondiabetic Dutch adults: genetic loci and clinical and lifestyle parameters, and their interactions in the lifelines cohort study. J Intern Med. 2013;273:283-93.

13. Prendergast C, Smyth O, Murray F, Cunningham SK, McKenna TJ. The relationship of blood glucose and haemoglobin A1 levels in diabetes subjects. Irish J Med Sci. 1994;163:233-5.

14. Rohlfing CL, Wiedmeyer H-M, Little RR, England JD, Tennill A, Goldstein DE. Defining the relationship between plasma glucose and HbA1c. Analysis the glucose profiles and $\mathrm{HbA1c}$ in the Diabetes Control and Complications Trial. Diabetes Care. 2002;25:275-78.

15. Sikaris K. The correlation of hemogobin A1c to blood glucose. J Diabetes Sci Technol. 2009;3:429-38.

16. Wei N, Zheng H, Nathan DM. Empirically establishing blood glucose targets to achieve HbA1c goals. Diabetes Care. 2014;37:1048-51.

17. Van't Riet E, Alssema M, Rijkelijkhuizen JM, Kostense PJ, Nijpels G, Dekker JM. Relationship between A1c and glucose levels in the general Dutch population. The New Hoorn Study. Diabetes Care. 2010;33:61-66.

18. Ketema EB, Kibret K. Correlation of fasting and postprandial plasma glucose with HbA1c in assessing glycemic control; systematic review and metaanalysis. Arch Public Health. 2015;73:43.

19. Monnier L, Lipinski H, Colette C. Contributions of fasting and postprandial plasma glucose increments to the overall diurnal hyperglycaemia of type 2 diabetic patients. Diabetes Care. 2003;26:881-5.

20. Walicka M, Jóźwiak J, Rzeszotarski J, et al. PROGENS-HbA $A_{1 c}$ study: safety and effectiveness of premixed recombinant human insulin (Gensulin M30). Arch Med Sci. 2016;12(5):985-91.
21. Guidelines on the management of diabetic patients. A position of Diabetes Poland. Clin Diabetol. 2017;6 (suppl A):A1-A79.

22. National Glycated Haemoglobin Standardisation Program. Harmonizing hemoglobin A1c testing. http://www.ngsp.org. Accessed 01 Oct 2016.

23. Marley JV, Oh MS, Hadgraft N, Singleton S, Isaacs K, Atkinson D. Cross-sectional comparison of pointof-care with laboratory HbA1c in detecting diabetes in real-world remote Aboriginal settings. BMJ Open. 2015;5:e006277.

24. Gialamas A, St John A, Laurence CO, Bubner TK, PoCT Management Committee. Point-of-care testing for patients with diabetes, hyperlipidemia or coagulation disorders in the general practice setting: a systematic review. Fam Pract. 2010;27:17-24.

25. Al-Ansary L, Farmer A, Hirst J, et al. Point-of-care testing for Hb1c in the management of diabetes: a systematic review and meta-analysis. Clin Chem. 2011;57:568-76.

26. Lenters-Westra E, Slingerland RJ. Three of 7 hemoglobin A1c point-of-care instruments do not meet generally accepted analytical performance criteria. Clin Chem. 2014;60(8):1062-72.

27. Herman WH, Cohen RM. Racial and ethnic differences in the relationship between HbA1c and blood glucose: implications for the diagnosis of diabetes. J Endocrinol Metab. 2012;97:1067-72.

28. Snieder H, Sawtell PA, Ross L, Walker J, Spector TD, Leslie RD. HbA1c levels are genetically determined even in type 1 diabetes: evidence from healthy and diabetic twins. Diabetes. 2001;50:2858-63.

29. Soranzo N. Genetic determinants of variability in glycated hemoglobin $\left(\mathrm{HbA}_{1 \mathrm{c}}\right)$ in humans: review of recent progress and prospects for use in diabetes care. Curr Diab Rep. 2011;11:562-9. 